\title{
Application of desktop human in the loop simulation to study air operations
}

\author{
P. Sheehan $^{a}$ and J. Mukerjee ${ }^{a}$ \\ ${ }^{\mathrm{a}}$ Defence Science and Technology Organisation, 506 Lorimer Street, Fishermans Bend, VIC 3207 \\ Email: paul.sheehan@dsto.defence.gov.au
}

\begin{abstract}
While supporting the 2006 North West Shelf capability trial, Defence Science and Technology Organisation (DSTO) staff observed a Northrop Grumman virtual trial in which a surveillance scenario involving a simulated RQ-4B Global Hawk unmanned aerial vehicle (UAV) was conducted. From this trial DSTO identified a requirement to utilise human-in-the-loop (HITL) simulation to support acquisition and inform the development of tactics and concept of operations (CONOPS) for Project AIR 7000.

Development of an indigenous HITL simulation capability received endorsement through consultation with the AIR 7000 S\&T Strategy Group and became the Synthetic Human-in-the-loop AIR 7000 Research Program (SHARP). The SHARP is now developed and managed by the Aerospace Capability Analysis (ACA) branch within the Joint and Operations Analysis Division (JOAD) of DSTO. The ACA has partnered with industry and used an agile development process to develop a mature, reconfigurable HITL simulation capability based on a distributed architecture and a standardised integration framework. The SHARP has become one of the analytical tools within an operations analysis process, which DSTO employs to assist the Australian Defence Force $(\mathrm{ADF})$ to:

- develop tactics and CONOPS;

- model maritime intelligence, surveillance, reconnaissance and response (MISRR) roles;

- perform capability assessment;

- conduct problem exploration;

- investigate human factors issues;

- model operator inputs for constructive simulation; and

- validate constructive simulation outputs.
\end{abstract}

The CONOPS and tactics development life cycle (CTDL), is one of the main processes by which the ACA branch conducts operations research. It is an iterative process that integrates HITL and constructive simulation. The HITL simulation is used in the phase of problem development, where human operators are exposed to the military problem context within a simulation environment. The HITL simulation functions as a forum for military operators and researchers to resolve the problem into specific tactical options and their variants. The set of tactics and respective variants become inputs for the constructive simulation phase which performs robust analysis using Monte Carlo techniques. The output from the constructive simulation is passed through HITL simulation to test the veracity. This paper reports on the application of the CTDL within the ACA branch and provides examples where the SHARP has served as the HITL simulation capability. The paper also provides a summary of the design features within the SHARP that are used to achieve an agile desktop HITL simulation capability.

Keywords: Human-in-the-loop, simulation, air operations, distributed architecture, agile 


\section{INTRODUCTION}

The Defence Science and Technology Organisation (DSTO) currently utilises human-in-the-loop (HITL) simulation to support acquisition and inform the development of concept of operations (CONOPS) and tactics. The benefits of HITL simulation were observed while supporting the 2006 North West Shelf capability trial, after which DSTO identified a requirement to develop an indigenous HITL simulation capability within the Aerospace Capability Analysis (ACA) branch of the Joint and Operations Analysis Division (JOAD). Subsequently the ACA branch partnered with industry and applied an Agile development process to establish the HITL simulation capability called the Synthetic Human-in-the-loop AIR 7000 Research Program (SHARP), to assist the Australian Defence Force (ADF) with:

- modelling maritime intelligence, surveillance, reconnaissance and response (MISRR) roles;

- performing capability assessments;

- conducting problem exploration;

- modelling operator inputs for constructive simulation; and

- validating output from constructive simulation.

This paper describes the approach utilised to develop the SHARP, where the system resides within a larger operations analysis (OA) process, and also provides examples in which, the SHARP has been used as a HITL simulation capability to assist the ADF.

\subsection{Background}

The major factor that led to the development of the SHARP was a simulated unmanned aerial vehicle (UAV) trial at Northrop Grumman's Cyber Warfare Integration Network (CWIN) facility in San Diego, California during October 2006 (Craig (2007)). During this trial it was evident that the benefits of HITL simulation were particularly significant for the work DSTO conducts in support of major acquisitions. The simulated CWIN trial was part of a larger activity to assess the maritime surveillance potential of the Globalhawk and Mariner UAVs to defend the North West Shelf environment.

The simulated trial modelled a UAV 'flown' by a crew comprised of a mission commander, a sensor operator and a pilot. A team of operations and human factors analysts were present to assess the realisation or otherwise of trial objectives and the utility of both the Global Hawk UAV against the operational aims, as well as evaluating the simulation system developed by Northrop Grumman. The trial provided an opportunity for exploration of new tactics and procedures in a wide range of scenarios and environmental conditions. The simulation demonstrated the potential utility of this HITL approach for addressing questions that concern the development of capability and CONOPS for maritime surveillance. It was evident to the DSTO staff supporting the trial that such a HITL simulation capability, which provides a flexible immersive environment to assist in the exploration of operator behaviour, would contribute to future studies in support of Project AIR $7000^{1}$.

\section{SHARP WITHIN THE CONOPS AND TACTICS DEVELOPMENT LifECYCLE}

The OA conducted within the ACA continues along the original purpose of operations research (OR) which is to support military decision makers (Crowther and Whiddington (1948)). The ACA specialises in supporting the operational effectiveness of military aircraft, either as individual platforms, or in a wider mission context. The two main purposes for conducting OA within the ACA are:

1. to contribute to the development of tactical and operational procedures; and

2. to provide advice to Australian Defence Force (ADF) when new platforms and systems are being considered for acquisition (Bennett et al. (2001)).

We aim to situate the HITL approach applied within the SHARP environment and locate its role within the CONOPS and tactical development lifecycle (CTDL) that is used within the ACA. The CTDL process has been described by authors using different labels in previous publications (Ibal et al. (2001); Bennett et al. (2001); Chandran et al. (2007); Heinze et al. (2002)). However, the purpose underpinning the process has remained constant, to strive for gains in operational performance through the review of CONOPS and tactics. The cyclic nature of the CTDL is one of the many persistent reminders that tactics need to be reviewed and adapted for

\footnotetext{
${ }^{1}$ www.defence.gov.au/publications/capabilityplan2012.pdf
} 
an operational environment that is always changing. A current illustration of the CTDL is presented in figure $1^{2}$.

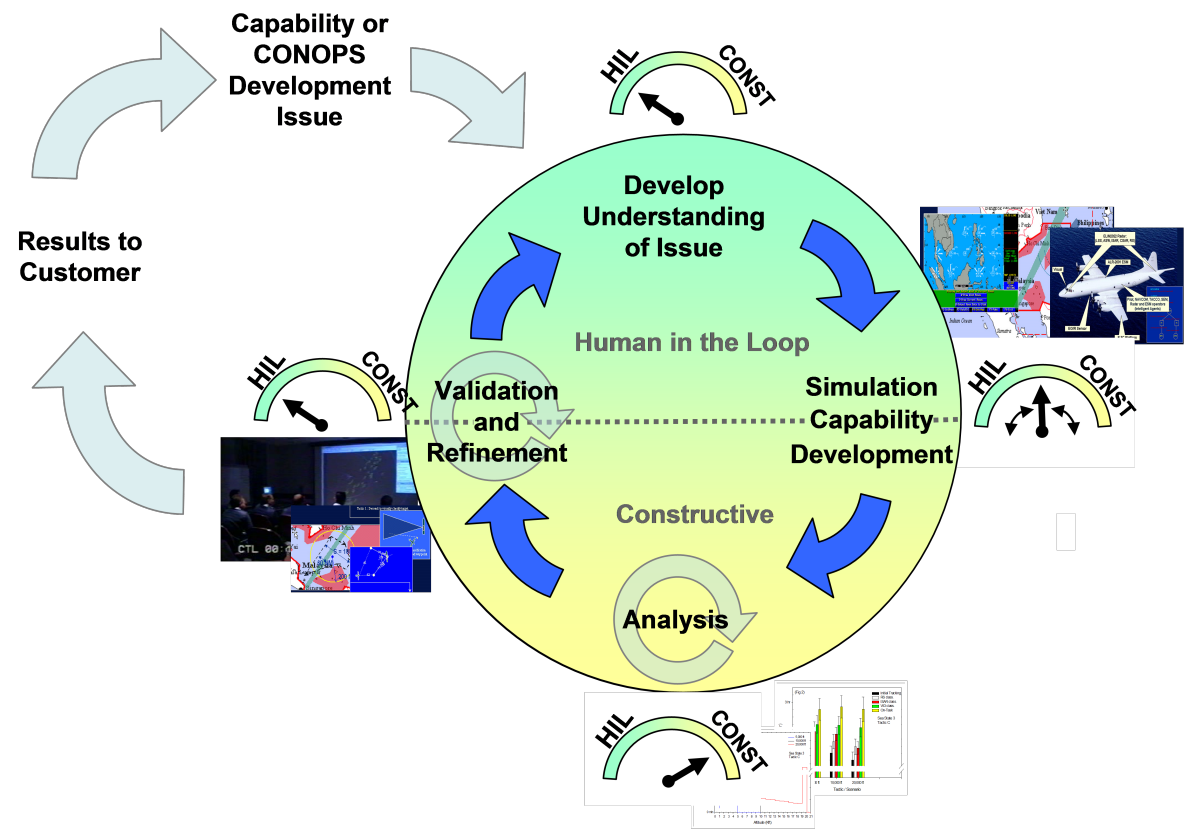

Figure 1. The CONOPS and tactics development lifecycle

The SHARP is an environment designed to apply HITL simulation for the exploration of military CONOPS and tactics. It is utilised to make a contribution in the areas labelled Develop Understanding of Issue and Validation and Refinement which in the CTDL (figure 1) has a need for military operator engagement. This engagement can be achieved using a number of methods such as meetings or workshops. The SHARP provides a HITL simulation capability that also serves as a forum for engagement between DSTO scientists and military operators. The application of the SHARP environment relies on the use of scenarios and exposing military experts to the simulated performance of proposed CONOPS and tactics in a structured manner, while DSTO analysts observe, document and record tactical decision making of the military experts (Heinze et al. (2002); Nilsson et al. (2008)). The exposure through simulation is enveloped by briefings, where structured discussion is used to review observed decisions. The briefings enable analysts to capture the reasoning applied by the experts to make the decisions that are observed during the simulation (Bankes (1992); Jakobsson et al. (2009)). Exploratory activities conducted through the SHARP has:

- investigated tactics and CONOPS for MISRR roles;

- identified data and model requirements for progression through the CTDL; and

- investigated human factors issues.

Simulation is applied in many forms across a number of domains, the application types range from full mission simulators to faster than real time (constructive). The aerospace domain, due to the mission critical nature of its operations, has a large footprint in the use and innovation of simulation products driven by a need to enhance safety and efficiency. The use of desktop simulation is not unique to the SHARP, rationale that underpins the design of the SHARP environment is similar to that of the Airspace Operations Laboratory (AOL) of the National Aeronautics and Space Administration, Ames Research Center (Prevot et al. (2006)). The AOL has been designed for studying air traffic management in the current environment as well as operational concepts for the Next Generation Air Transportation System. Desktop simulation has been used to study the effects of communication on workload and situation awareness at a tactical level (Cummings (2004)) and the relationship between the type of communication and workload within an Air Battle Management setting (Strang et al. (2012)).

\footnotetext{
${ }^{2}$ Developed by ACA team members Martin Cross, Luke Tracey and David Cox
} 


\section{SHARP ENVIRONMENT DESIGN}

A key decision made was to develop the SHARP within an Agile 'Scrum' framework (Schwaber (1997)) to ensure that it evolves as a flexible multi-purpose capability that can be altered relatively quickly to meet changing priorities. A Scrum is an instantiation of the Agile framework for producing software. It is an iterative software development process for managing product development, that is incremental and flexible. A key principle of the Scrum process is its recognition that during a project the customers can change their minds about what they want and need (often called 'requirements churn'), and that unpredicted challenges cannot be easily addressed in a traditional predictive or planned manner. As such, the Scrum process adopts an empirical approach, accepting that the problem cannot be fully understood or defined, focusing instead on maximizing the team's ability to deliver quickly and respond to emerging requirements. The Agile Scrum framework thus ensures a releasable product, with new capability, that is produced at regular intervals whilst sustaining a feedback loop from the users of the system. In order to achieve this it was decided to employ a distributed architecture within a standardised integration framework.

\subsection{DISTRIBUTED ARCHITECTURE and STANDARDISED INTEGRATION FRAMEWORK}

The use of Distributed Architecture ${ }^{3}$ has made it possible to use commercial software where appropriate, which provides the system with a high level of adaptability. Employing commercial software has also removed the cost and time of developing system components independently and meant that technical support can be provided by a third party rather than consuming internal resources. A distributed architecture has enabled pre-existing models of system components to be reused and updated as necessary and it has also equipped the SHARP with the ability to interface with other simulation systems. The use of a distributed architecture together with a standardised integration framework allows capability to be augmented or reduced as required without disrupting the larger system. Consequently the models of each system component can effectively function in isolation, which allows development and testing to be carried out independently (figure $2^{4}$ ).

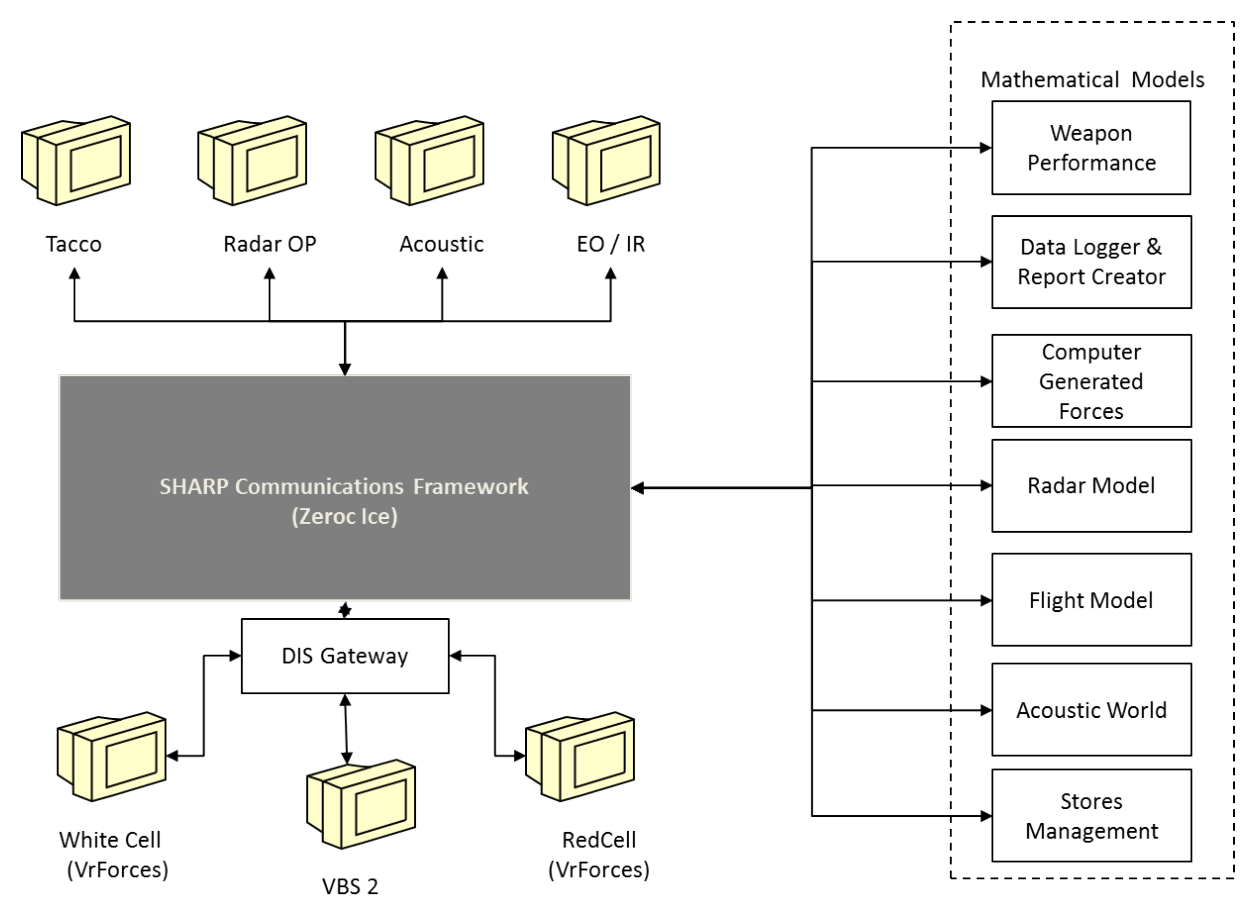

Figure 2. The SHARP Distributed Architecture

The modular design of the SHARP environment has resulted in a flexible system, which allows the rate of development effort to be governed by the Scrum process. This ensures that the rate of effort can be re-directed at short notice.

\footnotetext{
${ }^{3}$ www.sisostds.org/ProductsPublications/Standards/IEEEStandards.aspx

${ }^{4}$ Produced by Tim Patterson of Advanced VTOL Technologies, SHARP industry partner
} 


\subsection{THE SCRUM PROCESS}

The Scrum process structures development using cycles of effort called 'Sprints', which are sequential iterative periods of work that build on the previous iteration and they usually have two to four week durations. The Sprints are 'time boxed', which means they finish on a specific date regardless of whether or not the work has been completed, and are usually not extended. At the beginning of each Sprint, the development team (industry partners) uses customer (DSTO scientists) input to prioritise a set of requirements for completion. All other requirements exist in a list called the 'Product Backlog', which is reviewed programmatically and prioritised for selection in future Sprints. At the end of a Sprint, the team reviews the results with the customer and demonstrates the new capability that has been achieved. The Scrum process emphasises that the working product at the end of the Sprint is fully complete. In the case of software, this means a product that is integrated, fully tested, end-user documented and potentially usable by the end user of the system.

\section{HITL APPLICATIONS}

To date SHARP has been used as one of many analytical tools within the CTDL process. Two case studies of utilising SHARP by the ACA within an overarching CTDL process are now presented.

\subsection{RADAR TRACKING AUTOMATION}

This study used SHARP to explore the extent to which a radar auto-detect function assists operators to track surface targets by alerting the operator to potential targets appearing on their radar visual display. This Automatic-Track-While-Scan (ATWS) function automatically commences tracking high intensity returns without the need for operator intervention, which would reduce the need of operators to continually scrutinise their display for new contacts and manually initiate tracking. In the absence of this automation it is likely that operator workload would increase, affecting tactical operations, and decreasing the likelihood of achieving mission objectives.

Participants in the exercise included four experienced RAAF AP-3C Orion ${ }^{5}$ operators, who undertook the roles of pilot, tactical commander, radar operator and electro-optics operator. The scenario involved a general area of operations (AO) with a shipping lane comprising of fishing boats, merchant vessels of different types, and naval warships. Participants were rotated through scenarios with and without the ATWS feature and were required to detect and track as many surface targets as possible.

The study was able to provide an indication of the extent by which the ATWS feature was able to reduce operator workload and increase mission effectiveness. A more significant discovery was that it also qualitatively identified various human factors issues that impacted the benefits of the automation, which may have been missed without enveloping the HITL simulation by structured elicitation of the participant experience.

\subsection{ESTIMATION OF CLASSIFICATION RANGES}

This study used the SHARP to explore the typical ranges at which operators flying a helicopter at low altitudes are able to classify targets using both visual inspection and the Inverse Synthetic Aperture Radar (ISAR) mode of the radar. The ISAR mode utilises the motion along the length of a target to produce a high quality image, allowing an operator to classify the contact. During the study, operators were required to fly through a realistic $\mathrm{AO}$ in real time and attempt to classify all surface maritime contacts within the area. These classification ranges were then used within a constructive simulation model to model the approximate ranges at which an operator would be expected to make both a visual classification and a classification using the ISAR mode of the radar. The constructive simulation model was then used to explore and develop tactics and operations for helicopter operations.

\section{SUMMARY}

The ACA branch of the JOAD continues to produce output that serves as a Scientific aid to purposeful decisions (Stratton (1975)). The ACA is progressing the development of various elements of analytical capability that contribute to operationalise the CTDL. The ACA branch has successfully used HITL simulation to support Project AIR 7000 through the modelling of the ADF's MISRR roles, capability assessments and the investigation of human factors issues.

This paper has described the development of a HITL simulation tool, using an Agile (Scrum) approach, and

\footnotetext{
${ }^{5}$ http://www.airforce.gov.au/Technology/Aircraft/AP-3C-Orion/?RAAF-//YPMc68jYhKbVgHtZ92chiR88FVCnyo
} 
employed it within the CTDL process. The SHARP has been implemented using Distributed Architecture within a Standardised Integration Framework to ensure that it remains flexible in its development and was able to integrate specialised models where appropriate.

To date, the SHARP has primarily been used as a tool to explore the ADF's maritime surveillance problem space and to model operator decisions as a means to inform more detailed constructive analyses. This work has primarily existed within the Develop Understanding of Issue phase of the CTDL. However, future use of the SHARP will include employing it as a tool to test the veracity of the results from constructive simulations, particularly in support of the development of maritime surveillance tactics and CONOPS. This will ensure that DSTO is able to provide the ADF with a HITL simulation capability that will support maritime surveillance platforms during their life cycle through concept, acquisition and tactical employment.

\section{ACKNOWLEDGEMENT}

We would like to acknowledge the DSTO staff that have delivered this capability from a concept to operational, David Cox, Martin Cross and Luke Tracey and our industry partners Advanced VTOL Technologies and CAE Australia. The SHARP capability is funded by the Royal Australian Air Force and the Royal Australian Navy.

\section{REFERENCES}

Bankes, S. (1992). Exploratory modelling and the use of simulation for policy analysis. Technical report, RAND, Santa Monica, USA.

Bennett, K., T. Josefsson, S. Goss, M. Cross, S. Waugh, and T. Truong (2001). An Application of DSTO's Battle Model using Agents and Humans-in-the Loop. SimTecT Proceedings 2001, 99-110.

Chandran, A., G. Ibal, and Z. Tu (2007). Developing ARH Troop Tactics Using Operations Research Constructive Simulation.

Craig, D. (2007). North west shelf unmanned aerial system trial. Technical report, DSTO-TR-1958.

Crowther, J. G. and R. Whiddington (1948). Science at war. Philosophical Library.

Cummings, M. L. (2004). The need for command and control instant message adaptive interfaces: Lessons learned from Tactical Tomahawk human-in-the-loop simulations. CyberPsychology \& Behavior 7(6), 653661.

Heinze, C., S. Goss, T. Josefsson, K. Bennett, S. Waugh, I. Lloyd, G. Murray, and J. Oldfield (2002). Interchanging agents and humans in military simulation. AI Magazine 23(2), 37.

Ibal, G., Arney, A. M., Mockridge, L., and Murray, G. (2001). Air Operational Analysis in Support of Army Aviation. In Proceedings of the Land Warfare Conference.

Jakobsson, A, Bulluss, Gary and Watson, Jamie (2009). Multi-method synergies empower complex capability development. In Proceedings of INCOSE.

Nilsson, M., J. van Laere, T. Ziemke, and J. Edlund (2008, June). Extracting rules from expert operators to support situation awareness in maritime surveillance. In Information Fusion, 2008 11th International Conference on, pp. 1-8. IEEE.

Prevot, T., N. Smith, E. Palmer, J. Mercer, P. Lee, J. Homola, and T. Callantine (2006). The airspace operations laboratory (AOL) at NASA ames research center. In Proceedings of the AIAA Modeling and Simulation Technologies Conference.

Schwaber, K. (1997). Scrum development process. In Business Object Design and Implementation, pp. 117134. Springer.

Strang, A. J., S. Horwood, C. Best, G. J. Funke, B. A. Knott, and S. M. Russell (2012). Examining temporal regularity in categorical team communication using sample entropy. In Proceedings of the Human Factors and Ergonomics Society Annual Meeting, Volume 56, pp. 473-477. SAGE Publications.

Stratton, A. (1975). Operational research:(i) its origins and development in government service. Contemporary Physics 16(6), 575-588. 